\title{
EL CONCEPTO DE PUDOR EN KIERKEGAARD: ANÁLISIS DE LA DETERMINACIÓN SEXUAL EN EL CONCEPTO DE ANGUSTIA
}

\author{
Pablo Uriel Rodríguez \\ doi: 10.11144/Javeriana.uph36-72.cpks
}

\section{RESUMEN}

A pesar de la extraordinaria variedad de estudios sobre la filosofía de Kierkegaard, su concepto de pudor no ha recibido suficiente atención. Mi trabajo tiene como objetivo presentar y analizar el concepto de pudor de Kierkegaard, tal como se desarrolla en El concepto de angustia (1844) del seudónimo Vigilius Haufniensis. Primero, intento brindar una comprensión de algunos de los temas principales del libro de Haufniensis (espíritu, tarea, angustia). Segundo, examino lo que manifiesta el fenómeno psicológico del pudor sobre la estructura de la subjetividad del ser humano. Palabras clave: Kierkegaard; pudor; sexualidad; espíritu; intersubjetividad

\footnotetext{
Universidad de Morón, Universidad de Buenos Aires, Conicet y Universidad Nacional General, Sarmiento, Argentina.

Correo electrónico: blirius@hotmail.com

Para citar este artículo: Rodríguez, P. U. (2019). El concepto de pudor en Kierkegaard: análisis de la determinación sexual en El concepto de angustia. Universitas Philosophica, 36(72), 251-277. ISSN 0120-5323, ISSN en línea 2346-2426. doi: 10.11144/Javeriana.uph36-72.cpks
} 


\title{
THE CONCEPT OF MODESTY IN KIERKEGAARD: AN ANALYSIS OF THE SEXUATE CONDITION IN THE CONCEPT OF ANXIETY
}

\begin{abstract}
Despite of the extraordinary variety of studies on Kierkegaard's philosophy, his concept of modesty has not received enough attention. My paper aims to present and analyze Kierkegaard's concept of modesty, as it is developed in The Concept of Anxiety (1844) by his pseudonym Vigilius Haufniensis. First, I will try to provide an understanding of some of the major themes of Haufniensis's book (spirit, task, anxiety). Second, I examine what the psychological phenomenon of modesty reveals about the subjective structure of the human being.
\end{abstract}

Keywords: Kierkegaard; modesty; sexuality; spirit; intersubjectivity 


\section{Planteamiento de la cuestión}

EN EL AÑo 1943 el filósofo judeo-alemán Martin Buber publica Das Problem der Menschen. El libro en cuestión reelabora una serie de clases impartidas en el marco de un curso de verano consagrado a la antropología filosófica, que había tenido lugar cinco años antes en la Universidad Hebrea de Jerusalén. Hacia la mitad del primer capítulo de la segunda parte, Buber (1974) desliza la siguiente apreciación sobre la filosofía de Kierkegaard: "en Kierkegaard este hacerse 'singular' no es más que el supuesto para entrar en relación con Dios: solo después de haberse hecho 'singular' puede el hombre entrar en esta relación” (p. 99). Esta apreciación denota una posición ambigua frente a la obra del danés: de reconocimiento y, al mismo tiempo, de rechazo. Lo más valioso del pensamiento de Kierkegaard radica en el hecho de no concebir al "yo" en soledad, sino vinculado con un "tú", mientras que su principal déficit consiste en que, dentro del conjunto de las relaciones del individuo, únicamente el encuentro personal del "yo humano" con el "tú divino" posee un carácter esencial, siendo las restantes relaciones, en último término, prescindibles. Para la interpretación buberiana, el yo del que habla Kierkegaard se constituye a si mismo cerrándose ante los otros para luego abrirse únicamente a Dios. ¿Es atinada esta imagen del "singular" kierkegaardeano? Resulta evidente que la presentación de Buber responde de manera prácticamente exclusiva a una determinada, y quizás parcial, comprensión de la figura de Abraham en Temor y temblor (1994), publicado originalmente en 1843: deviene "singular" aquel individuo capaz de sacrificar los lazos que lo ligan con sus seres queridos para alcanzar una relación absoluta con lo Absoluto. Según esta interpretación, la tarea de "llegar a ser sí mismo" vendría a coincidir con un movimiento de individualización, esto es, con un proceso por el cual el yo construye su identidad a partir de la relación consigo mismo. En consecuencia, el otro -especialmente el otro humano- no jugaría rol esencial alguno en la constitución y el desarrollo de la personalidad ${ }^{1}$. Tradicionalmente se pensó que esta lectura era válida a la hora de describir el abordaje de la subjetividad desarrollado en lo que la crítica especializada denomina la primera autoría seudónima

1 Con esta perspectiva coincide, entre otros, Mark Taylor (1977, pp. 111-112) en su artículo "Love and Forms of Spirit: Kierkegaard vs. Hegel”. 
del danés -la serie de publicaciones que comienza en 1843 con O lo uno o lo otro (2006) y culmina en 1846 con la aparición del Postscriptum (2009)-².

Existen al menos dos motivos de peso que obligan a revisar el criterio de recepción del corpus kierkegaardeano mencionado en el párrafo anterior. En primer lugar, es necesario notar, junto a Patricia Dip (2012, p. 12) y Rafael Larrañeta (1990, p. 146), que el libro Las obras del amor (1965), de 1847, más que un nuevo inicio del filosofar kierkegaardeano, es el remate de una reflexión en torno al fenómeno erótico presente en $O$ lo uno o lo otro, de 1843 y Etapas en el camino de la vida (1952), de 1845. Las primeras publicaciones seudónimas ya se estarían ocupando del aspecto intersubjetivo de la personalidad a través de su tratamiento de la cuestión del amor ${ }^{3}$. En segundo lugar, cabe señalar que el autor seudónimo de La enfermedad mortal (1984), de 1849, describe al individuo en los términos de una doble relación, a saber, la que desarrolla consigo mismo y la que lo vincula con el otro. De esta manera, en el marco de esta comprensión del ser humano, un proceso existencial de individualización que se proponga como metas la separación y el aislamiento poseería, forzosamente, un valor negativo ${ }^{4}$. Por tanto, si se afirma que la caracterización del yo brindada por Anti-Climacus es la reflexión más profunda y articulada en torno al ser humano, entonces releer las consideraciones respecto a la formación de la personalidad presentes en las obras publicadas entre 1843 y 1846 bajo la luz de la teoría del yo elaborada en La enfermedad mortal permitiría hacer visible aquel aspecto social de la personalidad que parece faltar en los primeros libros firmados bajo seudónimo.

A la hora de presentar las conclusiones de su artículo titulado "Ethics as the Connection Between Subjectivity and Intersubjectivity”, Pia Søltoft (1998)

2 John Elrod (1980, p. 180), por citar un ejemplo paradigmático, piensa que Kierkegaard examina la función y el valor de las relaciones intersubjetivas en la formación del yo únicamente en sus obras tardías, comenzando en Las obras del amor y alcanzando su punto culminante en La enfermedad mortal.

3 Puede decirse que en el análisis del amor desarrollado en el libro de 1847, Kierkegaard conduce indirectamente una reflexión sobre el rol que juega el otro en la constitución del yo preguntando de qué modo el yo influye con su praxis amorosa en el desarrollo de la personalidad del prójimo (Kierkegaard, 1965).

4 En contra de lo planteado por Buber, para Anti-Climacus el yo no deviene "singular" para luego relacionarse con Dios, sino que es, precisamente, a través de su interacción con Dios como llega a ser "singular". 
afirma que "Kierkegaard posee una perspectiva dialógica moderada, en la cual el sí mismo no se constituye a través de la relación con el Otro, pero sí alcanza a través de dicha relación su continuidad, su autorrealización o su autoplenificación" (p. 69; traducción propia) ${ }^{5}$. El gran mérito de este trabajo es el de detectar, exponer y analizar este "dialoguismo moderado" no solo en los escritos de Kierkegaard posteriores a 1846, sino también, y con mayor énfasis, en sus primeros textos: Sobre el concepto de la ironía, de 1841, el segundo volumen de O lo uno o lo otro (2006), Temor y temblor (1994) y La repetición (1997), publicado en 1843. Resulta llamativo que entre los ejemplos aportados por la especialista danesa a favor de su hipótesis de lectura no figure El concepto de angustia, publicado el 17 de junio de 1844. Precisamente, el principal propósito del presente artículo es demostrar que en el texto del seudónimo Vigilius Haufniensis la dimensión intersubjetiva de la personalidad juega un rol significativo.

Centraremos nuestra mirada en el segundo capítulo del libro, "La angustia como pecado hereditario progresivamente considerado". Al igual que Gregory Beabout (1996), sostenemos que esta sección de El concepto de angustia, desde una perspectiva filosófica y psicológica, funciona como "una defensa contra la acusación de individualismo radical” (p. 58). El eje de nuestro argumento consiste en un análisis del concepto de pudor (Blufardighed en danés, Scham en alemán), una categoría que no ha recibido la suficiente atención por parte de los estudiosos de la obra del danés. Hemos optado por acotar el estudio de esta categoría a las alusiones que de ella se realizan en el libro publicado por el seudónimo Vigilius Haufniensis puesto que, como afirman Michael Theunissen y Wilfried Greve (1979, p. 30), la cualificación del hombre como pecador le permite al autor de El concepto de angustia examinar los temas antropológicos fundamentales de los primeros escritos seudónimos. La exposición se desarrollará en dos momentos: en primer término, ofrecemos una presentación sumaria del análisis psicológico de la angustia desarrollado por el seudónimo de turno ${ }^{6}$;

5 "Kierkegaard has a moderated dialogical view, where the Self is not constituted, but it gets its continuity, its self-realization or selfffulfillment in the relation to the Other".

6 Por razones de espacio prescindimos de una reconstrucción global de la categoría de angustia y nos concentramos en aquellos aspectos que, a nuestro entender, tienen una vinculación directa con el interés particular del presente escrito, a saber, el concepto de pudor. 
en segundo lugar, precisamos uno de los aspectos esenciales de la estructura de nuestra subjetividad que se hace visible a partir de la experiencia del pudor, a saber, el carácter sexuado del yo humano ${ }^{7}$. Por último concluimos con una serie de apreciaciones en torno a la relación entre sexualidad y amor.

\section{Espíritu, síntesis e historia: el discurso psicológico de la angustia}

El discurso psicológico de Vigilius Haufniensis se propone desarrollar una reflexión en torno a la realidad del ser humano a partir de un análisis de la experiencia vital de la angustia. Dicho con las palabras de Arne Grøn (2008), "lo que a Kierkegaard le interesa no es la angustia en tanto que estado aislado de la psiquis, sino aquello que la angustia revela sobre el hecho de ser un ser humano" (p. 2; traducción propia) ${ }^{8}$. Cuando, al comienzo del capítulo final de El concepto de angustia, el seudónimo escribe "si un hombre fuera un animal o un ángel, no podría angustiarse" (El concepto de angustia $[C A], 26 ; S K S 4,454)^{9}$, afirma con ello que la angustia es un fenómeno exclusivamente humano y que, como tal, pone de manifiesto aspectos antropológicos esenciales.

El hombre, dice Vigilius Haufniensis en el último capítulo de su obra, es capaz de angustiarse porque es una síntesis (Synthese). La explicación más extensa y articulada del significado de la palabra sintesis como definición del ser humano y su relación con el fenómeno de la angustia aparece en el quinto apartado del primer capítulo ${ }^{10}$ :

7 Por razones de extensión, limitamos la presente investigación a las primeras experiencias del pudor y excluimos de nuestro trabajo el análisis de la función que cumple el pudor durante el acto sexual.

8 "What interests Kierkegaard is not anxiety as an isolated state of mind but what anxiety reveals about being a buman being."

9 Para las citas de Kierkegaard utilizamos las traducciones al castellano indicadas en las Referencias. Ofrecemos también la paginación de la última edición de sus obras completas, indicando en números arábigos tanto el volumen como la página: Søren Kierkegaard Skrifter [SKS].

10 Dejaremos de lado el análisis de la síntesis en relación con la temporalidad, desarrollado por el danés en el tercer capítulo de El concepto de angustia. El lector interesado puede consultar el artículo "Spirit and Temporality in The Concept of Anxiety" de Grøn, 2001. 
El hombre es una síntesis de lo anímico [det Sjelelige] y de lo corporal [det Legemlige]. Pero una síntesis es impensable si los dos no se unifican en algo tercero. Lo tercero es el espíritu [Aand]. En la inocencia, el hombre no es meramente un animal, pues si fuera meramente un animal en un momento cualquiera de su vida, entonces nunca se volvería hombre. El espíritu, entonces, está presente, pero como algo inmediato, como soñando. Claro que, en cuanto está presente, es de alguna manera un poder hostil, pues está siempre perturbando la relación entre el alma y el cuerpo, relación que persiste, ciertamente, y que sin embargo no tiene persistencia sino desde el momento en que la obtiene del espíritu. Por otro lado, es un poder amistoso que quiere justamente constituir la relación. ¿Cuál es entonces la relación del hombre con ese poder ambiguo, cómo se relaciona el espíritu consigo mismo y con su condición? Se relaciona como angustia ( $C A, 161 ; S K S$ 4, 349).

Un análisis de este fragmento, que pretenda ser integral y coherente, no puede dejar de enfrentarse con serias dificultades. ¿Qué hay que entender cuando se dice cuerpo (Legeme), alma (Sjel) y espíritu (Aand)? La comprensión de lo corporal no reviste mayores inconvenientes; con este término el seudónimo hace referencia a aquellos aspectos físicos y materiales de la realidad humana que se encuentran en permanente devenir. Las cosas comienzan a complicarse a la hora de fijar el sentido de la palabra alma. Por lo menos en el contexto específico del pasaje transcripto, Vigilius Haufniensis realiza un empleo técnico del sustantivo Sjel, que no posee connotaciones estrictamente religiosas. Alma sirve aquí para aludir a la dimensión mental del ser humano, esto es, al registro de las operaciones intelectuales ${ }^{11}$. Sin embargo, hablar del alma como lo mental no alcanza a agotar el espectro semántico cubierto por dicho término. Jesús Antonio Collado (1962, pp. 32-33) ofrece una original traducción capaz de capturar una faceta esencial de la cuestión: Sjel debe entenderse como 'vida' o, más precisamente, como 'principio vital'. La audacia del intérprete español es sumamente productiva

11 John Elrod (1975, pp. 38-39) distingue tres usos del término alma en Kierkegaard. En primer lugar, en un sentido religioso; en segundo lugar, como equivalente del 'yo' o 'sí mismo' y, en tercer lugar, lo que él distingue como su uso técnico, como sinónimo de las facultades mentales del yo y, esencialmente, como 'imaginación'. Cabe señalar que el análisis de Elrod se funda en La enfermedad mortal y supone la identidad entre los pares de opuestos cuerpo-alma y finito-infinito. 
porque da cuenta del estrecho vínculo entre lo psíquico (alma) y lo físico (cuerpo). Por tanto, la primera conclusión que puede extraerse de la elucidación de estos conceptos es que el autor de El concepto de angustia intenta dejar atrás cierta concepción dualista del hombre. El ser humano es un compuesto psicosomático. Cuerpo y alma pueden distinguirse y separarse en el plano del análisis lógico, pero permanecen unidos en el plano de la realidad. A partir de esta convicción, el seudónimo será capaz de sostener enfáticamente que las distorsiones que afectan al individuo ni son exclusivamente corporales ni exclusivamente anímicas; por este motivo, según el autor seudónimo, cuando alguna de estas esferas de lo humano entra en conflicto, las consecuencias de esta desorganización (Desorganisation) repercuten en la otra y viceversa $(C A, 231 ; \operatorname{SKS} 4,423)^{12}$.

Tras caracterizar lo corporal y lo anímico, resta considerar al espíritu. Sin lugar a dudas, la comprensión de este elemento implica las mayores dificultades. Lo primero que salta a la vista en torno al espíritu es su carácter activo: el espíritu puede ser definido como un "agente sintetizador de los diferentes compuestos de la síntesis” (Taylor, 1974, p. 86) ${ }^{13}$. Dicha actividad, no obstante, está signada por cierta ambigüedad. El espíritu, y únicamente él, es capaz de estabilizar (poner positivamente) pero también de desestabilizar (poner negativamente) la unidad entre el cuerpo y el alma: la presencia del espíritu abre una grieta hacia el interior del compuesto sintético ${ }^{14}$. A partir de su irrupción será únicamente su mediación la que haga posible [o no] la relación entre lo corporal y lo anímico. La segunda cuestión a analizar exige tomar nota de dos afirmaciones presentes

12 El ideal existencial perfilado por Vigilius Haufniensis precisa de una plenitud tanto a nivel corporal como mental. Sobre esta cuestión, véase Rodríguez, 2013a, pp. 83-95.

13 El espíritu como "tercero" no es una entidad diferente que se añada a las dos previas (cuerpo y alma), sino una función (Nordentoft, 2009, pp. $45-46$ y Grøn, 2001, p. 136). Para una interpretación sustancial del espíritu, puede consultarse Kierkegaard y Unamuno. La existencia religiosa de Collado (1962, pp. 32-33, nota al pie).

14 El análisis de Vigilius Haufniensis presupone la distinción esbozada por el seudónimo estético A en la primera parte de $O$ lo uno o lo otro. El compuesto psicosomático puede ser pensado desde dos perspectivas: 1) con un carácter inmediato, en tanto la determinación última es anímica y la articulación entre los dos elementos es armoniosa, o 2) con un carácter mediato, en tanto la determinación última es espiritual y la articulación entre ambos elementos del compuesto se halla en tensión (OO, 84-85; SKS 2, 68-69). 
en El concepto de angustia: i) que la relación del hombre con el espíritu es la relación del espíritu consigo mismo y ii) que esta autorrelación debe ser entendida en los términos de la relación del espíritu con su poder, esto es, la experiencia que el espíritu hace de sí mismo en calidad de principio activo. El espíritu se relaciona consigo mismo al relacionarse con los elementos de la síntesis; en el pasaje aludido, se nos dice de manera explícita e inequívoca que esta relación es la angustia. Que el espíritu se vincule con su propia actividad a través de la angustia significa que la vivencia que aquel tiene de sí es, al igual que su poder, de carácter ambiguo: el espíritu quiere tanto aferrarse a sí mismo como deshacerse de sí mismo. Al experimentar angustia, dirá el seudónimo en el tercer capítulo de su obra, el espíritu se relaciona consigo mismo "como algo externo" ( $C A$, 208; SKS 4, 399). Si la relación del ser humano con el espíritu es equiparada con la relación del espíritu consigo mismo, entonces aquello que se diga del espíritu en relación consigo mismo se dirá, a su vez, de la relación del ser humano consigo mismo. De esta coincidencia entre el espíritu y el ser humano se sigue, como sostiene Grøn (2000, p. 29), la no coincidencia del ser humano consigo mismo: la angustia nos revela que el ser humano, siendo una síntesis, se experimenta a sí mismo como otro.

Al hablar de la extrañeza del ser humano ante sí mismo nos adentramos en una última cuestión de capital importancia dentro del planteamiento del seudónimo. Tanto en el tercer como en el cuarto capítulo de El concepto de angustia, el verbo bare es empleado para describir la función del espíritu en relación a la síntesis. La palabra danesa puede traducirse como 'sostener', 'llevar' o 'transportar'. Resulta claro que Vigilius Haufniensis no pretende referirse a un acontecimiento puntual, sino, más bien, a un proceso continuo; dicho de otro modo, a una acción que se prolonga en el tiempo y que debe renovarse a cada momento. Cuando "el espíritu se pone a sí mismo (Aanden satter sig selv) -dice el seudónimo-, pone la síntesis (satter den Synthesen)" (CA, 165; SKS 4, 354) y, de allí en adelante, la unidad entre el cuerpo y el alma solo posee persistencia, sea cual sea su signo, en la medida en que "la obtiene del espíritu" ( $C A, 161 ; S K S$ 4, 349). El equilibrio entre cuerpo y alma, bajo la presencia del espíritu, es de carácter dinámico; la unidad entre los elementos de la síntesis humana debe ser producida en todo momento porque también en todo momento puede quebrarse. Conquistar dicha armonía, siempre amenazada, es la constante tarea (Opgave) del espíritu. 
Adelantemos, por el momento, que la tarea del espíritu es la resolución de una contradicción (Modsigelse). Ahora bien, de acuerdo con el seudónimo, la resolución de toda contradicción exige un movimiento y este movimiento recibe la denominación de historia (Historie).

La autoposición del espíritu es a su vez la posición de su tarea, por lo cual la "historia del espíritu" se identifica con la "historia de la tarea del espíritu"; esto permite especificar el contenido del discurso psicológico desarrollado por Vigilius Haufniensis. Siguiendo en mayor medida la terminología de Karl Rosenkranz que la de Hegel, la "Introducción" de El concepto de angustia define la psicología como "doctrina del espíritu subjetivo" $(C A, 143 ; S K S 4,331)^{15}$. Al margen de las innegables discrepancias entre Kierkegaard, por un lado, y Hegel y Rosenkranz, por el otro, existe entre ellos un punto fundamental en común: para todos estos pensadores la doctrina del espíritu subjetivo es una filosofía del espíritu finito, esto es, un análisis de las facultades (intelectuales, emocionales y voluntarias) del individuo humano ${ }^{16}$. Ahora bien, si el espíritu no es otra cosa más que su actividad y esta actividad es su historia, entonces el discurso psicológico como doctrina del espíritu subjetivo se resuelve, en definitiva, en la descripción de la historia del espíritu y, con ello, en la descripción de la historia de la tarea del espíritu. La psicología, como sostiene Darío González (2003, p. 377), es el nombre

15 Hegel reserva el término psicología para designar el último momento de su filosofía del espíritu subjetivo. La doctrina hegeliana del espíritu subjetivo se completa con la antropología y la fenomenología. La identidad entre psicología y doctrina del espíritu subjetivo está tomada a partir del título del libro Psicología o ciencia del espiritu subjetivo (Psychologie oder die Wissenschaft vom subjectiven Geist, Königsberg, 1837) del hegeliano Karl Rosenkranz. La ciencia del espíritu subjetivo de Rosenkranz está estructurada en tres secciones: para las dos primeras secciones conserva la denominación hegeliana, antropología y fenomenología; la última sección recibe el nombre de pneumatología. Para un análisis de la influencia de Rosenkranz en Kierkegaard, se puede consultar el artículo de Heiko Schulz (2016) "Rosenkranz: Traces of Hegelian Psychology and Theology".

16 La principal divergencia es que el danés considera que a diferencia de la psicología de Hegel, la suya es una filosofía del espíritu finito, no solo en el sentido objetivo, sino también subjetivo del genitivo. En este sentido, el seudónimo estético de $O$ lo uno o lo otro no pretende definir la esencia de la "conciencia desdichada", sino describir qué significa ser un desdichado (OO, 234; SKS 2, 216). Kierkegaard repite una y otra vez que la psicología es una disciplina que debe limitarse al análisis de un territorio determinado, a saber, el del espíritu subjetivo, y que, por tanto, debe evitar desarrollarse como ciencia del espíritu absoluto. 
con el cual los seudónimos designan aquella teoría que expone la progresiva constitución y el funcionamiento de la subjetividad humana ${ }^{17}$.

¿Cuál es el valor que el discurso psicológico le asigna al fenómeno de la angustia? Contestar esta pregunta equivale a dilucidar qué papel juega la angustia en la historia del espíritu. El autor seudónimo es claro al respecto: el hecho de que el espíritu experimente angustia señala que su historia sigue abierta y su tarea inconclusa. La presencia de la angustia indica que el espíritu no ha llegado a sí mismo, es decir, que el espíritu no ha logrado establecer la síntesis, eliminando por completo la contradicción. Desde un punto de vista formal la tarea del espíritu es siempre la misma: acceder a sí mismo determinando la síntesis. No obstante, si es posible hablar de historia es porque tanto el espíritu como el contenido de su tarea no permanecen inalterados: por una parte, la tarea se modifica en la medida en que la actividad del espíritu reconfigura progresivamente la síntesis; por otra parte, dado que el espíritu se relaciona consigo mismo relacionándose con la síntesis, el devenir mismo de la síntesis implica un devenir del espíritu. Así como el espíritu y la tarea que este debe realizar poseen una historia, es lógico pensar que también la posee la angustia. Según el pensamiento de Vigilius Haufniensis, "puesto que [el ser humano] es una síntesis, puede angustiarse" (CA, 261; SKS $4,454)^{18}$; en consecuencia, es posible sostener que una variación en el equilibrio de la síntesis provoca una variación en la angustia. Dos cuestiones se imponen a partir de lo dicho. Primera: puesto que toda relación del espíritu consigo mismo, mientras su tarea no se haya consumado, es una relación de angustia, la historia del espíritu y la historia de la angustia se desarrollan en paralelo. Segunda: las diferentes figuras o modalidades de la angustia estudiadas a lo largo de El concepto de angustia pueden ser pensadas como momentos de la historia de la angustia.

17 En la línea de las diversas "historias de la subjetividad" desarrolladas por los idealistas alemanes, la psicología de Kierkegaard no es un estudio de facultades y capacidades estáticas, sino una investigación sobre la génesis y el desarrollo de dichas facultades. Es necesario, ante todo, señalar que la teoría desarrollada por los seudónimos no quiere hacer foco en una noción universal de subjetividad: el sujeto del cual pretende dar cuenta el análisis psicológico no es la instancia universal de la autorreferencia, esto es, el Yo del idealismo alemán, sino esa individualidad única e irrepetible que se encarna en una existencia singular ( $C A, 248-249 ; S K S 4,443)$.

18 En relación con esta idea, Walter Schulz (1979) sostiene que "la angustia es auténtica solo cuando no es una 'pura angustia espiritual'” (p. 353). 
El breve análisis precedente tuvo un carácter propedéutico; su objetivo fue el de esclarecer un marco de comprensión para el fenómeno del pudor. Por este motivo, antes de abordar el objeto específico del presente estudio, resulta conveniente explicitar los conocimientos que en referencia a nuestro tema ya hemos ganado con los párrafos anteriores. Dado que, de acuerdo con las palabras del seudónimo (CA, 183, 185; SKS 4, 372, 375), el fenómeno del pudor es una manifestación particular de la angustia, podemos dejar establecidas las siguientes características en torno a su naturaleza. Como toda variante de la angustia, el pudor es:

i) Una relación del espíritu consigo mismo y, por ello, en él se revela una modalidad particular del comportamiento del ser humano en referencia a su propio ser.

ii) Una posición asumida por el espíritu frente a la síntesis psíquicocorporal. En tanto que tal, es "saber" de una contradicción y, con ello, “determinación” de un aspecto específico de la tarea.

iii) Un momento en la historia del espíritu. Por tanto, acompaña determinados eventos y circunstancias de la vida humana.

\section{El concepto de pudor: el espíritu ante la sexualidad}

Si el Ser humano, en tanto QUe espíritu, no estuviese orientado hacia sí mismo, sería incapaz de experimentar pudor ${ }^{19}$. $\mathrm{Al}$ afirmar que la vivencia del pudor "puede despertarse a sí misma" ( $C A, 183 ; S K S$ 4, 372-373), Vigilius Haufniensis establece que dicho fenómeno encuentra su condición suficiente y necesaria en la estructura autorrelacional del ser humano. En alusión a este pasaje, el fenomenólogo francés Michel Henry (2001) concluye que el pudor se suscita en el yo "sin que sea necesaria [...] la intervención de una mirada extraña" (p. 258). El comentario de Henry posee un gran valor porque ilumina la diferencia fundamental entre el enfoque del seudónimo kierkegaardeano y la perspectiva sartreana. Si bien la vergüenza (bonte) nos habla de una relación íntima del

19 "El pudor es la expresión más concreta de lo que hace humano al hombre y lo diferencia del animal, esto es, la capacidad de relacionarse consigo mismo" (Nordentoft, 2009, p. 62, traducción propia). ["Modesty is the most concrete expression of that which makes man into man and differentiates him from the animal, namely, the ability to relate himself to himself"]. El pudor es, como lo describe Till Breyer (2006, p. 8), un afecto reflexivo. 
yo consigo mismo, lo cierto es que, para el autor de El ser y la nada, un examen exclusivo y exhaustivo del "ser-para-si" no arroja indicio alguno de dicha vivencia: "la vergüenza no es originariamente un fenómeno de reflexión [...] [puesto que], en su estructura primera, es vergüenza ante alguien” (Sartre, 1998, p. 291). Sin la presencia efectiva del prójimo, específicamente de su mirada (regard), el yo no podría avergonzarse de sí mismo ${ }^{20}$. Esta referencia a la conceptualización sartreana de la vergüenza nos permite plantear un interrogante fundamental que pone en cuestión nuestra tesis: ¿en qué sentido cabe hablar de intersubjetividad en la teoría del yo desarrollada en El concepto de angustia, si en la experiencia del pudor descripta por Vigilius Haufniensis el ser humano se avergüenza de sí mismo ante sí mismo?

Para comenzar a contestar esta cuestión resulta conveniente volver a confrontar la exposición del seudónimo con otra perspectiva, en este caso con las alusiones hegelianas al sentimiento de pudor (Schamhaftigkeit) presentes en la sección de las Lecciones sobre la estética consagrada al estudio de la vestimenta en la escultura. Para el filósofo alemán, el pudor es una autoconciencia de carácter crítico que traduce afectivamente cierto reproche que el individuo dirige contra sí mismo. En palabras del propio Hegel (1989):

[...] el hombre que deviene consciente de su determinación superior a ser espíritu debe considerar lo solo animal como una inadecuación y tratar de ocultar primordialmente las partes de su cuerpo, tronco, pecho, espalda y piernas, que desempeñan funciones meramente animales (p. 542).

20 Sumamente clarificador es el comentario de Michael Theunissen (2013): "la estructura formal de la vergüenza atestigua al otro como la condición individual y concreta de mi ser. Porque el Yo del que me avergüenzo es, antes que nada, puesto por el otro ante quien me avergüenzo. En tanto me quedo solo, no puede surgir en mí la vergüenza, porque no solo falta él frente a quien siento vergüenza, sino también falta él de quien puedo tener vergüenza. Yo tengo que ser visto por el otro para que pueda experimentar vergüenza, esto es, para avergonzarme de quien el otro ve. Esta es la peculiaridad del fenómeno de la vergüenza: que el objeto de mi vergüenza no lo soy yo como existiendo para mí mismo, sino yo en cuanto soy para el otro, y por eso constituido por él [...]. La vergüenza no es un fenómeno reflexivo y no puede ser tal cosa porque la presencia del otro, expresada en la determinación 'ante', no puede reconciliarse con la actitud reflexiva [...]. El Yo de quien me avergüenzo no es el que existe para mí, sino el que se aparece al otro” (p. 237). 
El pudor funciona como una señal de alarma: la incomodidad que el individuo siente al exponer su cuerpo manifiesta la posibilidad de un retroceso desde la esfera del espíritu hacia la esfera de la naturaleza. Cabe marcar, por tanto, dos coincidencias fundamentales entre las Lecciones y El concepto de la angustia. En primer lugar, en ambos textos el pudor es concebido como un fenómeno esencialmente reflexivo. En segundo lugar, tanto Hegel como el seudónimo kierkegaardeano consideran que el pudor implica una "comprensión" anímica de una de las funciones esenciales del espíritu: la configuración (determinación) espiritual de la corporalidad humana. Pese a estas semejanzas, la caracterización que Haufniensis hace del pudor contiene una particularidad que lo distingue claramente del discurso hegeliano. De acuerdo con Hegel, el sentimiento de pudor manifiesta la extrañeza del espíritu ante la corporalidad en general. En el capítulo 411 de la Enciclopedia de las ciencias filosóficas, el filósofo alemán afirma que en la totalidad de su figura el cuerpo es "algo inmediato y natural y, por ello, solo puede ser un signo indeterminado y enteramente imperfecto del espíritu, y no es capaz de representarlo como es para sí mismo como universal" (Hegel, 2010, p. 468). El discurso psicológico del seudónimo kierkegaardeano dota de un contenido más específico al sentimiento de pudor: el espíritu siente angustia puesto que "no solo está determinado como cuerpo, sino como cuerpo con una distinción de género” ( $C A, 183 ; S K S$ 4, 372). El pudor, en suma, es la reacción del espíritu ante el hecho de tener que configurar un "cuerpo sexuado".

Según El concepto de angustia, el del espíritu con el cuerpo sexuado es un vínculo entre dos realidades completamente heterogéneas. Abstraído de su relación con el cuerpo, el espíritu es un poder indeterminado, lo que quiere decir que en sí mismo el espíritu es ajeno a cualquier facticidad o característica concreta y, por ello mismo, "en el espíritu no hay diferencia ${ }^{21}$ alguna entre varón y mujer” (CA, 185; SKS 4, 374) $)^{22}$. El cuerpo, por su parte, es la sede de toda una

21 A lo largo de los dos primeros capítulos de su libro, Vigilius Haufniensis utiliza tres expresiones para dar cuenta de la distinción entre lo masculino y lo femenino: sexuelle Forskjellighed (diversidad sexual), sexuelle Differents (diferencia sexual) y generiske Differents (diferencia genérica). La distinción conceptual contemporánea entre sexo y género no se explicita en ningún momento del texto.

22 El espíritu humano no es un espíritu puro; desde el inicio, es un espíritu encarnado. Por este motivo, cabría interpretar la indiferencia sexual del espíritu no como una oposición radical a la 
serie determinaciones que permiten la individualización del ser humano. Sin embargo, la determinación sexual es diferente al resto de las determinaciones con las cuales debe relacionarse el espíritu al entrar en contacto con el cuerpo, puesto que, como escribe Haufniensis, solamente "lo sexual es la expresión de la enorme contradicción (Wiederspruch) que consiste en que el espíritu inmortal está determinado como genus [género]" (CA, 184; SKS 4, 373). Al entrar en contacto con un cuerpo sexuado, el espíritu padece un proceso de sexuación. Debe lidiar, entonces, con una realidad que, por una parte, le resulta ajena a su ser y completamente inexplicable; pero que, por otra parte, se le ha vuelto propia ${ }^{23}$. Para clarificar la relación del espíritu con lo sexual, Walter Dietz (1994, p. 114) utiliza la palabra alemana verstrickt, con la que traduce el término danés hildet: el espíritu se encuentra 'trabado' en su sexualidad ${ }^{24}$. Yendo más allá de Dietz, podemos entender esta expresión en un doble sentido. En primera instancia, en un sentido peyorativo: la sexualidad implica una limitación de carácter negativo para el espíritu. No obstante, en segunda instancia, puede pensarse también en un sentido constitutivo: la sexualidad es una condición del espíritu humano y, como tal, es un polo orientador de su comportamiento.

Hacia el final del punto anterior quedó establecido que el fenómeno del pudor, siendo una modalidad específica de la angustia, se hace presente en un momento determinado de la historia del espíritu. De lo que se trata, ahora, es de definir cuál es este momento en contraposición al que lo antecede y al que lo precede; en definitiva, se trata de distinguir diversas posiciones del espíritu ante lo sexual. Las primeras alusiones en torno a la sexualidad aparecen en dos párrafos del apartado 6 del primer capítulo.

Dejaremos de lado los antojos de los proyectistas y supondremos con toda simplicidad la existencia de la diferencia sexual antes de la caída, mencionando

sexuación, sino como cierta neutralidad plástica originaria: que el espíritu no sea ni masculino ni femenino significaría que puede ser tanto lo uno como lo otro y que esta posibilidad en ningún momento se cancela.

23 La misma idea ya había sido anticipada hacia el final del primer capítulo del libro: "solo en la sexualidad se pone la síntesis como contradicción" ( $C A, 166 ; S K S 4,354)$.

24 Dietz hace referencia a una bella expresión empleada por Vigilius Haufniensis $(C A, 166 ; S K S 4$, 354): "hildet Fribed" (libertad trabada o, como traduce Óscar Parcero, libertad impedida). 
tan solo que no la había, puesto que no se da en la ignorancia. En esto contamos con el respaldo de la Escritura.

En la inocencia, Adán era, en tanto que espíritu, un espíritu que sueña. La síntesis, por tanto, no es real, pues lo vinculante es justamente el espíritu, y este no está puesto todavía como espíritu. En el animal, la diferencia sexual puede estar desarrollada de manera instintiva, pero en el hombre, justamente porque es una síntesis, no puede darse de esa manera. En el momento en que el espíritu se pone a sí mismo, pone la síntesis [...]. Antes de ese tiempo, no es un animal, pero tampoco es propiamente un hombre [...] (CA, 165; SKS 4, 354).

El tratamiento de la sexualidad desarrollado por Vigilius Haufniensis comienza desestimando lo que Jean Libis denomina escatología andrógina. El filósofo francés explica que el Nuevo Testamento sugiere que "la adscripción a una determinación sexual está destinada a desaparecer al final de los tiempos” (Libis, 2001, p. 140). Ahora bien, en la época de Kierkegaard, el pensamiento romántico alemán proyectó la superación de la sexualidad del fin de los tiempos al inicio de la historia del mundo: la neutralidad sexual futura es, en definitiva, una restauración de la auténtica y más primitiva naturaleza humana. El autor seudónimo de El concepto de angustia piensa que el cuerpo humano está determinado desde un principio como cuerpo masculino o cuerpo femenino y, por este motivo, rechaza de plano la idea romántica de que "la falta original instauró la vergüenza del cuerpo sexuado e introdujo una distancia dramática entre el hombre y la mujer -que anteriormente formaban 'una sola carne"” (Libis, 2001, p. 140). Pese a ello y a su manera, el discurso psicológico de Haufniensis conserva algo de esa indistinción sexual originaria postulada por los románticos ${ }^{25}$. Adán y Eva se diferencian sexualmente desde un punto de vista estrictamente anatómico; no obstante, esta diferencia no tiene un significado claramente definido

25 El mismo Kierkegaard expresa esta idea romántica a través de uno de sus más famosos seudónimos, Johannes el Seductor, el personaje principal de Diario del seductor, el escrito que culmina el primer volumen de $O$ lo uno o lo otro $(O O, 430 ; S K S$ 2, 432). Sobre la figura del "andrógino original" en Kierkegaard, puede consultarse "El mito del andrógino original en Kierkegaard" (Rodríguez, 2013b). 
para ellos ${ }^{26}$ y, en este sentido, como afirma Susana Munnich (1986), "no existe como actualidad" (p. 177). Desde un punto de vista psicológico, puede decirse que antes de la irrupción del espiritu la distinción sexual no diferencia plenamente a los seres humanos entre sí. Ahora bien, que la irrupción del espíritu (la actividad inicial de la libertad, en términos teológicos, el "primer pecado") sea, al mismo tiempo, el establecimiento de la sexualidad significa que cuando los seres humanos experimentamos nuestra sexualidad, vivenciamos un fenómeno que rebasa el registro de la corporalidad.

El hombre -comenta al respecto Collado (1962)- no puede considerar lo sexual como un fenómeno aislado, sino que ha de conocer su trascendencia en el conjunto de la personalidad y decidir por sí mismo la función que ha de desempeñar en su vida (p. 116).

En este sentido, el seudónimo kierkegaardeano estima que la sexualidad posee una gran importancia y significación para la vida del ser humano y, por este motivo, la cuestión concerniente a lo sexual requiere de un tratamiento teórico urgente. Por otra parte, Haufniensis ( $C A, 182 ; S K S$ 4, 371) considera que este tratamiento, por serlo de un fenómeno que rebasa la animalidad y se constituye como específicamente humano, únicamente será adecuado en la medida en que sea desarrollado dentro de los límites de una filosofía del espíritu subjetivo o psicología ${ }^{27}$.

El autor seudónimo de El concepto de angustia utiliza el término ignorancia para definir el modo en que el espíritu, que todavía no ha sido enteramente establecido, se vincula con la diferencia sexual. Ignorancia no es aquí sinónimo de completo desconocimiento ${ }^{28}$; sino, más bien, una inquieta y ambigua relación con aquella marca anatómica, la de una particularidad, que todavía no posee un

26 "La distinción de género está puesta en la inocencia, pero no está puesta como tal" ( $C A, 193$; SKS 4,383).

27 Es posible interpretar estas afirmaciones como una crítica velada al hecho de que Hegel, en la Enciclopedia de las ciencias filosóficas, se ocupa de la sexualidad en el marco de su "Filosofía de la naturaleza" (2010, \$\$369 y 370 ).

28 Para Vigilius Haufniensis la "ignorancia propiamente dicha [...] le está reservada solo al animal” (CA, 183; SKS 4, 372). 
sentido claramente definido. Esta ignorancia, como toda angustia, es referencia a un misterioso objeto que atrae y repele, es decir, relación con "algo" que en su indeterminación se presenta como una "nada"29. Al mismo tiempo, la ignorancia que embarga al espíritu en esta etapa prerreflexiva es, como señala Rudi Visker (2005, p. 249), la incomprensión de cuál es su tarea en relación a esta nada que le es dada.

La angustia, al igual que el espíritu y la libertad, posee una historia y, por lo tanto, está sujeta a cambios. ¿Qué modificación padece la angustia a lo largo de su devenir histórico? De acuerdo con Vigilius Haufniensis, "es como si la nada que es el objeto de la angustia se volviera más y más un algo” (CA, 177; SKS 4, 366). Se trata, por tanto, de una alteración en aquello a lo cual la angustia se refiere: un pasaje de la indeterminación a la determinación. En el caso de la angustia de la sexualidad, por lo dicho, este progreso traduce una mayor comprensión de la diferencia sexual por parte del espíritu. El siguiente pasaje expone dos momentos sucesivos de esta "historia de la angustia sexual" que deberemos abordar para elucidar el significado del pudor:

[...] el pudor es un saber acerca de la distinción de género, pero no a la manera de una relación con la distinción de género, es decir, el impulso no está presente como tal [...]. En el pudor está puesta la distinción de género, pero no en relación con su otro. Esto sucede en el impulso (CA, 183; SKS 4, 372-373).

Comencemos analizando el más tardio de estos dos momentos, aquel cuya categoría central es el impulso. Vigilius Haufniensis distingue expresamente entre instinto (Instinkt) e impulso (Driften). El primer término parece ser utilizado para hacer referencia a una tendencia animal; el segundo término, por el contrario, se reservaría para aludir a una tendencia humana, esto es, a una tendencia con algún grado de conocimiento y, por tanto, de alguna manera, libre ${ }^{30}$. El impulso, por tanto, es un saber no teórico - pero paradójicamente lúcido y preciso- de la

29 Sobre la relación entre la nada y la angustia habla Vigilius Haufniensis en los primeros párrafos del apartado 5 del capítulo inicial de su libro (véase $C A$, 159-161; SKS 4, 347-349).

30 Valga mencionar que, de acuerdo con el autor seudónimo de El concepto de angustia (CA, 183; SKS 4, 372), el instinto animal es ciego y, por tanto, carente de conciencia, la acción animal no es propiamente libre. 
distinción de género, puesto que, de acuerdo con la teoría psicológica del seudónimo, en él se expresa la relación de un sexo con el otro. Queda resuelto el enigma de la sexuación del cuerpo que tanto inquietaba al espíritu: con el impulso se expone la particularidad de lo masculino como orientación erótica a lo femenino y la particularidad de lo femenino como orientación erótica a lo masculino. Este "secreto" solo puede conocerlo un espíritu que ya se ha puesto a sí mismo y, al mismo tiempo, ha establecido su relación con la síntesis del cuerpo y el alma; por este motivo, Vigilius Haufniensis, recurriendo al lenguaje religioso, dice que "cuando se probó el fruto del árbol del conocimiento, vino la distinción entre el bien y el mal, pero también vino la diferenciación sexual en tanto impulso" ( $C A$, 190; SKS 4, 380). Pero este saber de la distinción genérica presente en el impulso no lo puede conquistar el espíritu en soledad: el yo conoce el significado de la diferencia sexual únicamente a través de una conciencia provocada por la presencia concreta del otro, esto es, por el fenómeno del impulso.

¿Qué significado posee la diferencia sexual antes de que se haga presente el impulso? Resolver este interrogante equivale a determinar el sentido de ese segundo momento de la "historia de la angustia sexual" del cual nos hablaba el pasaje anteriormente transcripto, esto es, fijar el significado del pudor. Vigilius Haufniensis sostiene que, a diferencia de lo que ocurre en la experiencia del impulso, en la vivencia del pudor "no hay huella alguna de deseo sensual" (CA, 183; SKS 4, 372). A partir de ello, el seudónimo psicológico concluye que el origen del pudor no puede ser el deseo, sino que la angustia del pudor se despierta a símisma. Mientras que el impulso es una reacción afectiva (un deseo) suscitada en el yo (el individuo deseante) a partir de la presencia de otro (el individuo deseado), el pudor es una reacción afectiva que se suscita en el yo en presencia de sí mismo. Sin embargo, aquello que el yo sabe de sí mismo a través de la experiencia del pudor hace que el yo interrumpa su relación consigo mismo y se trascienda. La clave para entender esta trascendencia está contenida en la siguiente afirmación del seudónimo: "en el pudor está puesta la distinción de género, pero no en relación con su otro" ( $C A$, 183; SKS 4, 373). El espíritu pudoroso ya es consciente de su sexuación, pero sin poseer de esta determinación una comprensión precisa. La ausencia de un sentido definido, sin embargo, no significa la carencia de sentido; más bien hay que hablar de una anticipación o prefiguración de un sentido para la determinación genérica. Esta conciencia oscura de sí tendría su causa última en el hecho de que 
el yo pudoroso se relaciona exclusivamente consigo mismo y no con otro, mas esa relación del yo consigo mismo que es el pudor consistiría, justamente, en el presentimiento del hecho de que el yo está determinado a una relación con otro: al autorrelacionarse, al volverse sobre sí mismo y descubrir la sexuación de su propia realidad, el individuo vislumbra que su ser no se agota en la referencia a sí mismo. En el pudor, como en toda modalidad de la angustia, el yo experimenta (sabe) que no existe una unidad inmediata consigo mismo: él comprende que no se basta a sí mismo por ser, en tanto que individuo sexuado, un ser finito. Para el observador externo, el fenómeno del pudor permite apreciar que, siempre heterogéneo y dispar consigo mismo, e incluso "antes" de cualquier contacto con otro concreto, el yo está atravesado en lo más íntimo de su ser por una apertura a los otros ${ }^{31}$. El pudor expresa un descentramiento afectivo del yo: la relación del individuo consigo mismo se interrumpe por la referencia a un otro que, pese a su ausencia fáctica, se hace presente en la vivencia de la propia finitud. Hay, por decirlo de algún modo, una anticipación del otro a partir del yo; pero esta prefiguración del otro se constituye a través de aquello que en el yo es signo de su esencial incompletitud. El yo es, al mismo tiempo, autorrelacional (para sî) y alterrelacional (para otro): está referido a sí mismo y, a su vez, está referido al otro. Doble condición estructural de la subjetividad que Vigilius Haufniensis ( $C A, 148$; SKS 4, 335) condensa en su célebre fórmula: el ser humano es símismo y la especie.

\section{Balance final}

LLEGADOS A ESTE PUNTO, es necesario retomar algunos interrogantes que, de una forma u otra, hemos rozado a lo largo de nuestra exposición: ¿qué significa para el espíritu ser un espíritu sexuado? y ¿qué tarea le compete al ser humano en tanto que espiritu sexuado? Estos interrogantes formulados desde el marco del discurso psicológico (El concepto de angustia) encuentran su respuesta en las meditaciones en torno a la praxis amorosa cristiana (Las obras del amor).

31 Interpretando especulativamente el texto del seudónimo, podemos caracterizar el impulso (la tendencia afectiva de cada uno de los sexos al otro) como una orientación derivada. Así las cosas, la alterrelacionalidad como estructura originaria del espíritu tendría la plasticidad suficiente como para soportar otros tipos de orientaciones (véase la nota 25 , supra). 
La sexualidad, sostiene el autor de El concepto de angustia, es "el extremo de lo sensual" ( $C A, 165 ; S K S 4,354)$. Lo que el seudónimo kierkegaardeano quiere señalar con esta enigmática expresión es que, entre todas las necesidades y apetencias físicas y psicológicas que afectan al individuo, ninguna como el deseo sexual, sea cual sea su orientación, influye tan activa y profundamente en su conducta y existencia. Sin embargo, el discurso psicológico nos dice al mismo tiempo que el espiritu sexuado carga con una contradicción: siendo esencialmente indeterminado, el espíritu recibe con la sexualidad una determinación y, de ese modo, se torna extraño para sí mismo. Junto con la contradicción se establece la tarea de resolverla. Una empresa que coincide con la esencia misma del espíritu: devenir sí mismo. Ahora bien, dado que el espíritu humano es espiritu sexuado, el ser bumano debe llegar a ser-si mismo en este ser-otro:

[...] debo abstenerme de sacar cualquier conclusión desacertada, como, por ejemplo, que entonces la verdadera tarea sería hacer abstracción, es decir, en sentido extrínseco, aniquilar lo sexual. Una vez que lo sexual está puesto como extremo de la síntesis, ninguna abstracción sirve de nada. La tarea, naturalmente, consiste en hacerlo entrar en la determinación del espíritu ( $C A$, 193; SKS 4, 383).

Vigilius Haufniensis se siente en la obligación de dejar bien claro que la sexualidad en sí misma no debe ser rechazada: "lo sexual en cuanto tal no es lo pecaminoso" ( $C A, 183 ; S K S 4,372)$ y "lo sexual no es pecaminosidad" ( $C A, 192$; $S K S 4,382)$. La sexualidad que sí repudia es aquella que, por obra del egoísmo, se ha independizado por completo de lo espiritual. Con todo, el fundamento último de esta condena no es moral, sino psicológico y antropológico: una sexualidad constituida como un poder autónomo ${ }^{32}$ fractura la unidad vital a la cual está llamado todo ser humano. Muy lejos de proponer que el espíritu resuelva la

32 Cómo la sexualidad ha devenido una esfera autónoma dentro de la realidad humana, de qué manera el deseo erótico despierta y se desarrolla hasta lograr constituirse en el polo orientador de la conducta del hombre y qué papel jugó el cristianismo en este proceso, son cuestiones que Kierkegaard, desde una perspectiva estética, aborda y responde en "Los estadios eróticos inmediatos, o lo erótico musical" de la primera parte de $O$ lo uno o lo otro. Para un agudo análisis de este escrito en relación a los pasajes citados de El concepto de angustia, puede consultarse el trabajo de Nordentoft, 2009, pp. 53-65. 
contradicción excluyendo completamente lo erótico, el seudónimo kierkegaardeano entiende que la sexualidad posee un significado fundamental en la constitución del espíritu. El espíritu debe asumir y conducir todas las particulares posibilidades que se le abren al yo por el hecho de estar sexualmente determinado; no hacerlo implicaría desatender un campo de ejercicio abierto a su libertad. Vigilius Haufniensis designa la transfiguración de la sensualidad como una "victoria del amor (Kjerligheds Seier)" (CA, 193; SKS 4, 383). El amor, entonces, es el medio a través del cual la sexualidad se incorpora a la esfera del espíritu, una integración que debe ser contabilizada entre las obras del amor, algo de lo cual el mismo Kierkegaard deja constancia en sus meditaciones sobre el amor de 1847:

[...] el cristianismo está muy lejos de querer imprudentemente soliviantar la sensualidad contra el hombre mismo, a fuerza de enseñarle una espiritualidad exagerada [...]. Entiende por sensualidad algo distinto de eso que se suele llamar así, sin más ni más, y por eso de la misma manera que no les prohíbe a los hombres el que coman y beban, así tampoco se ha llamado a escándalo a propósito de un instinto que el hombre, desde luego, no se ha dado a sí mismo. Por lo sensual y lo carnal, el cristianismo entiende lo egoísta (OAI, 116; SKS 9, 59).

La vivencia que el espíritu realiza de su carácter sexuado implica un saberse a si mismo referido a otro: el yo humano es autorrelacional y alterrelacional. En este sentido, la teoría del pudor de 1844 anticipa uno de los puntos capitales del análisis de la subjetividad contenido en el tratado de la desesperación de 1849: el yo, dice el seudónimo Anti-Climacus, es "una relación que se relaciona consigo misma y que, relacionándose consigo misma, se relaciona a un otro” ( $E M, 36 ; S K S$ $11,130)^{33}$. Es preciso, no obstante, marcar una diferencia fundamental entre ambos escritos psicológicos ${ }^{34}$ : en El concepto de angustia, la alterrelacionalidad

33 Traducción ligeramente modificada.

34 Definida como teoría de la progresiva constitución y funcionamiento de la subjetividad del individuo humano, la psicología kierkegaardeana se expone, fundamentalmente, en El concepto de angustia y La enfermedad mortal. En el primer libro se exponen los momentos iniciales de la formación del yo, y en el segundo se hacen evidentes las consecuencias de ese proceso inicial y los intentos por modificarlas. Sin embargo, mientras que la investigación sobre la angustia se define como una investigación sencillamente "psicológica”, la investigación sobre la desesperación se concibe como 
es un elemento propio del espíritu, en tanto este es un espíritu encarnado; en $L a$ enfermedad mortal se convierte en una nota propia del espíritu sin más ${ }^{35}$. Volviendo al escrito de Vigilius Haufniensis, podemos decir que la doble estructura relacional del espíritu explica una suerte de solidaridad esencial del ser humano: cada individuo tiene la misma perfección [ser sí mismo y la especie], y es por eso que los individuos no se separan numéricamente unos de otros, como tampoco el concepto de la especie resulta ser un fantasma. Cada individuo está esencialmente interesado en la historia de todos los demás individuos, e incluso tan esencialmente como en la suya propia. Por eso la perfección es en sí misma la perfecta participación en el todo. Ningún individuo es indiferente a la historia de la especie, como tampoco lo es la especie a la del individuo (CA, 148; SKS 4, 335).

El "autointerés" no es en sí mismo una actitud pecaminosa; solo deviene tal cuando se configura como egoísmo, esto es, como un "interés en sí mismo" que prevalece por sobre el "interés en el otro". También es un desafío del amor llevar a su plenitud al carácter alterrelacional del yo humano; pero esta tarea, a decir de Kierkegaard, únicamente puede ser realizada por un amor edificado a partir de bases cristianas. Leemos en Las obras del amor (OAI, 118; SKS 9, 60) que todas las variedades y grados del amor no-cristiano tienen en común el ser una orientación afectiva hacia un "segundo yo". De acuerdo con la perspectiva del cristianismo, incluso "ese abandono con que el amante se entrega al único ser que ama, adhiriéndose a él con todas sus fuerzas" (OAI, 119; SKS 9, 61) no es más que un modo refinado de egoísmo: una reducción del otro al yo. "El amante -subraya Kierkegaard- no hace propiamente sino relacionarse a sí mismo egoístamente en

una investigación de "psicología cristiana". El concepto de angustia intenta pensar el problema del pecado a través de un fenómeno vital (la angustia) característico del ser humano: su punto de partida es la subjetividad humana como condición de posibilidad del pecado. La enfermedad mortal, por su parte, piensa la subjetividad humana a partir de un fenómeno vital (la desesperación): en la medida en que ese fenómeno vital es equiparado con el pecado, su punto de partida es el pecado como condición de la constitución de la subjetividad.

35 Nos limitamos a señalar que es objeto de discusión entre los especialistas en la filosofía del danés la siguiente cuestión: ¿̇la alterrelacionalidad, como estructura esencial de la subjetividad en La enfermedad mortal, se entiende en referencia a un otro específico, esto es, a Dios, o a cualquier otro? Véase Evans, 1997 y Walsh, 1987. 
esa entrega avasalladora e ilimitada" (OAI, 120; $S K S$ 9, 62); mas, de este modo, el equilibrio entre la autorrelación y la alterrelación queda destruido. El cristianismo enseña que el único amor auténtico es el que tiene como destinatario al "prójimo". ¿Quién es el prójimo? "El prójimo es 'el primer tú" (OAI, 123; SKS $9,64)$ : un otro que no está constituido a la medida del yo, sino conforme a su propia medida. Amando al otro como prójimo, el yo sitúa al otro al margen de todas sus expectativas y necesidades: deja al otro ser tro $^{36}$. Que el tú llegue a sersi-mismo en su relación con el yo constituye la mitad de la tarea del amor, puesto que a través de su relación con el tú también el yo debe llegar a ser sí mismo. El "interés en el otro" no debe anular el "interés en sí mismo":

[...] el prójimo es el que te está más próximo que todos los demás. ¿̇ncluso más próximo a ti que tú mismo? De ninguna manera, sino que cabalmente estará, o debe estarlo, tan próximo como tú lo estás de ti mismo [...]. Teniendo conciencia de ser dos, no hay posibilidad de ser un "yo" en el sentido egoísta de la palabra (OAI, 69; SKS 9, 29).

La verdadera libertad, esto es, la realización plena del espíritu, es, con la misma intensidad y dedicación, atención cuidadosa del otro (amor al prójimo) y atención cuidadosa del yo (amor a sí mismo).

Referencias

Beabout, G. (1996). Freedom and its Misuses: Kierkegaard on Anxiety and Despair. Milwaukee: Marquette University Press.

Breyer, T. (2006). Scham als Angst. Vergleichende Untersuchung zu Kierkegaards Entwurf eines Schambegriffs. E-Journal Philosophie der Psychologie, 5, 1-14.

Buber, M. (1974). ¿Quées el hombre? (Trad. E. Imaz). Ciudad de México: Fondo de Cultura Económica.

36 Francesc Torralba (2016), comentando la meditación kierkegaardeana en torno al amor, resume correctamente el objetivo del amor auténtico: "El amor no tiene como finalidad convertir al otro en una prolongación de sí mismo, sino que se pone a su servicio para que llegue a ser lo que, interiormente, está llamado a ser. El amor no limita ni mutila el proyecto de cada ser, sino que, en la medida en que quiere el bien ajeno, se somete a él" (p. 423). 
Collado, J. (1962). Kierkegaard y Unamuno. La existencia religiosa. Madrid: Gredos.

Dietz, W. (1994). Selbstverhältnis und Gottesverhältnis bei Augustin und Kierkegaard. Kierkegaardiana, 17, 109-129.

Dip, P. (2012). Teoría y praxis en Las obras del amor. Un recorrido por la erótica kierkegaardiana. Buenos Aires: Editorial Gorla.

Elrod, J. (1975). Being and Existence in Kierkegaard's Pseudonymous Works. Princeton: Princeton University Press.

Elrod, J. (1980). Kierkegaard on Self and Society. Kierkegaardiana, 11, 178-196. Evans S. (1997). Who Is the Other in Sickness Unto Death? God and Human Relations in the Constitution of the Self. En: N. Cappelørn \& H. Deusser (Eds.). Kierkegaard Studies. Yearbook 1997 (pp. 1-15). Berlin: Walter de Gruyter.

González D. (2003). Sin, Absolute Difference. En: N. Cappelørn, J. Stewart \& H. Deusser (Eds.). Kierkegaard Studies. Yearbook 2003 (pp. 373-383). Berlin-New York: Walter de Gruyter.

Grøn, A. (2000). The Human Synthesis. En: P. Houe, G. Marino \& S. Hakon Rossel (Eds.). Anthropology and Authority: Essays on Søren Kierkegaard (pp. 27-32). Amsterdam-Atlanta: Editions Rodopi.

Grøn, A. (2001). Spirit and Temporality in The Concept of Anxiety. En: N. Cappelørn \& H. Deusser (Eds.). Kierkegaard Studies. Yearbook 2001 (pp. 128-140). Berlin: Walter de Gruyter.

Grøn, A. (2008). The concept of Anxiety in Søren Kierkegaard. (Trad. J. B. L. Knox). Macon: Mercer University Press.

Hegel, G. W. F. (1989). Lecciones sobre la estética. (Trad. A. Brotons Muñoz). Madrid: Ediciones Akal.

Hegel, G. W. F. (2010). Enciclopedia de las ciencias filosóficas. (Trad. R. Valls Plana). Madrid: Alianza Editorial.

Henry, M. (2001). Encarnación: una filosofía de la carne. (Trad. J. Teira, G. Fernández \& R. Ranz). Salamanca: Ediciones Sígueme.

Kierkegaard, S. (1952). Etapas en el camino de la vida. (Trad. J. Castro). Buenos Aires: Santiago Rueda Editores.

Kierkegaard, S. (1965). Las obras del amor. Meditaciones cristianas en forma de discursos. Primera parte [OAI]. (Trad. D. G. Rivero). Madrid: Guadarrama. 
Kierkegaard, S. (1984). La enfermedad mortal [EM]. (Trad. D. G. Rivero). Madrid: Sarpe.

Kierkegaard, S. (1994). Temor y temblor. (Trad. V. S. Merchan). Barcelona: Altaya.

Kierkegaard, S. (1997). La repetición. (Trad. K. Hjelmström). Buenos Aires: JVE Psiqué.

Kierkegaard, S. (1997-2009). Søren Kierkegaard Skrifter (55 Vols.) [SKS]. N. J. Cappelørn, J. Garff, J. Kondrup, A. McKinnon \& F. H. Mortensen (Eds.). Copenhague: Søren Kierkegaard Forskningscenteret-Gads Forlag.

Kierkegaard, S. (2006). O lo uno o lo otro I. Fragmento de vida [OO]. Escritos de Søren Kierkegaard (Vol. 2/1). (Trad. D. González \& B. Saez Tajafuerce). Madrid: Trotta.

Kierkegaard, S. (2009). Postscriptum no cientifico y definitivo a Migajas filosóficas. (Trad. N. Bravo Jordan). Ciudad de México: Universidad Iberoamericana.

Kierkegaard, S. (2016). El concepto de angustia [CA]. Escritos de Søren Kierkegaard (Vol. 4/2) (pp. 125 - 278). (Trad. D. González \& O. Parcero). Madrid: Trotta.

Larrañeta, R. (1990). La interioridad apasionada. Verdad y amor en Søren Kierkegaard. Salamanca: Universidad Pontificia Editorial San Esteban.

Libis, J. (2001). El mito del andrógino. (Trad. M. Tabuyo y A. López). Madrid: Ediciones Siruela.

Munnich, S. (1986). Kierkegaard y la muerte del padre humano y divino. Santiago de Chile: Editorial Universitaria.

Nordentoft, K. (2009). Kierkegaard's Psychology. (Trad. B. H. Kirmmse). Eugene: Wipf \& Stock.

Rodríguez, P. (2013a). Salud vital o salud espiritual. De El concepto de la angustia a La enfermedad mortal. El arco y la lira, 1, 83-95.

Rodríguez, P. (2013b). El mito del andrógino original en Kierkegaard. Protrepsis. Revista de filosofia, 4, 45-67.

Sartre, J. P. (1998). El ser y la nada. (Trad. J. Valmar). Buenos Aires: Editorial Losada.

Schulz, W. (1979). Die Dialektik von Geist und Leib bei Kierkegaard. Bemerkungen zum Begriff Angst. En: M. Theunissen \& W. Greve (Eds.). 
Materialen zur Philosophie Søren Kierkegaards (pp. 347-366). Frankfurt am Main: Suhrkamp.

Schulz, H. (2016). Rosenkranz: Traces of Hegelian Psychology and Theology. En: J. Stewart (Ed.). Kierkegaard and his German Contemporaries (Vol. II). Theology (pp. 161-196). New York: Routledge.

Søltoft, P. (1998). Ethics as the Connexion Between Subjectivity and Intersubjectivity. Enrahonar, 29, 59-70.

Taylor, M. (1974). Kierkegaard on the Structure of Selfhood. Kierkegaardiana, 9, 84-103.

Taylor, M. (1977). Love and Forms of Spirit: Kierkegaard vs Hegel. Kierkegaardiana, 10, 95-116.

Theunissen, M. (2013). El otro. Estudios sobre la ontología social contemporánea. (Trad. G. G. Voet de Keyser). Ciudad de México: Fondo de Cultura Económica.

Theunissen, M., \& Greve, W. (1979). Einleitung: Kierkegaards Werk und Wirkung. En: M. Theunissen \& W. Greve (Eds.). Materialen zur Philosophie Soren Kierkegaards (pp. 9-104). Frankfurt am Main: Suhrkamp.

Torralba, F. (2016). La esencia del amor en Kierkegaard. Interpretación de Las obras del amor (1847). Pensamiento, 271, 411-429.

Visker, R. (2005). Demons and the Demonic. Kierkegaard and Heidegger on Anxiety and Sexual Difference. En: The Inhuman Condition. Looking for Difference After Levinas and Heidegger (pp. 235-254). United States of America: Kluwer Academic Publishers.

Walsh, S. (1987). On "Feminine" and "Masculine" Forms of Despair. En: R. L. Perkins(Ed.) InternationalKierkegaard Commentary. The Sickness unto Death. Volume 19 (pp. 121 - 134). Georgia: Mercer University Press. 